\title{
Analytical expressions for the flame front speed in the downward combustion of thin solid fuels and comparison to experiments
}

\author{
Toni Pujol ${ }^{1}$ and Bruna Comas ${ }^{2}$ \\ ${ }^{1}$ Departament d'Enginyeria Mecanica i de la Construccio Industrial, Escola Politecnica Superior, Universitat de Girona, \\ Campus Montilivi, E-17071 Girona, Catalonia, Spain \\ ${ }^{2}$ Departament de Fisica, Escola Politecnica Superior, Universitat de Girona, Campus Montilivi, E-17071 Girona, Catalonia, Spain
}

(Received 19 April 2011; revised manuscript received 15 July 2011; published 8 August 2011)

\begin{abstract}
We derive analytical expressions for the propagation speed of downward combustion fronts of thin solid fuels with a background flow initially at rest. The classical combustion model for thin solid fuels that consists of five coupled reaction-convection-diffusion equations is here reduced into a single equation with the gas temperature as the single variable. For doing so we apply a two-zone combustion model that divides the system into a preheating region and a pyrolyzing region. The speed of the combustion front is obtained after matching the temperature and its derivative at the location that separates both regions. We also derive a simplified version of this analytical expression expected to be valid for a wide range of cases. Flame front velocities predicted by our analytical expressions agree well with experimental data found in the literature for a large variety of cases and substantially improve the results obtained from a previous well-known analytical expression.
\end{abstract}

DOI: 10.1103/PhysRevE.84.026306

PACS number(s): 47.70.Pq, 47.70.Fw, 82.33.Vx

\section{INTRODUCTION}

A wide variety of systems in classical and interdisciplinary physics are modeled by reaction-convection-diffusion equations with wave front solutions that propagate at a constant velocity [1]. Several techniques have been developed in order to estimate such a speed, even for highly nonlinear reaction terms such as those found in combustion phenomena [2].

Simple burning processes of premixed gaseous fuels may follow a single one-dimensional reaction-diffusion equation for the gas temperature from which analytical estimates of the flame front speed may be derived [3]. These expressions have been recently generalized for more elaborate models described by coupled reaction-convection-diffusion equations for the gas temperature and fuel density by means of reducing the system to a single one-dimensional reaction-convectiondiffusion equation [4].

Flaming combustion of solid fuels, however, is a substantially more complex process involving several twodimensional coupled reaction-convection-diffusion equations in two major steps [5]. The first one consists of an endothermic chemical process where the solid loses weight and releases volatiles. The second one is an exothermic chemical process where the flammable gases of the volatiles react with atmospheric oxygen and produce combustion. The leading edge of the flame acts as the heat source for preheating and pyrolyzing the solid and also as the heat source for igniting the fuel gas mixture, which allows the flame front to propagate [6].

Here we study the flame spread in the downward combustion of thin solid fuels. These types of solids correspond to those with a characteristic thermal length larger than its half-thickness $\delta_{s}$. A well-known analytical expression for estimating the flame front velocity for this problem in a quiescent environment and in the limit of high activation energies for chemical reactions was derived by deRis [7], being

$$
v_{\mathrm{deRis}}=\frac{\pi}{4} \frac{\lambda_{g}}{c_{s} \delta_{s} \rho_{s \infty}} \frac{\left(T_{f}-T_{v}\right)}{\left(T_{v}-T_{\infty}\right)}
$$

where $T_{f}, T_{v}$, and $T_{\infty}$ are the flame, vaporization, and room temperatures, respectively, with $\lambda_{g}$ as the gas mixture conductivity evaluated at the reference temperature $\left(T_{f}+T_{\infty}\right) / 2, c_{s}$ is the solid specific heat, and $\rho_{s \infty}$ is the density of the virgin solid material.

Equation (1) was derived from reaction-convectiondiffusion equations by assuming a flame touching the surface with no fuel in front of it and no oxidizer behind it, a vaporizing solid at constant temperature, an Oseen flow, a unit Lewis number, and by neglecting buoyancy effects and conduction through the solid phase [8]. The flame front speed of deRis' model Eq. (1) produced results that reasonably agree with experimental data and combustion simulations [8]. However, Bhattacharjee et al. [9] have recently shown that Eq. (1) (excluding the $\pi / 4$ term) may follow from a simplified theory based on an energy balance applied to a control volume of the solid phase only. This opens a question: may we derive an analytical expression for the flame front speed focusing on the gas phase instead of on the solid one? Such an expression will, in principle, include some relevant effects ignored in Eq. (1) like the chemical kinetic ones for the gas phase. Our purpose has been to investigate the feasibility of deriving such an expression.

More precisely, the procedure for obtaining this expression has consisted of reducing the system of coupled reactionconvection-diffusion equations for gas temperature, oxygen mass fraction and fuel mass fraction, and of reaction-diffusion equations for solid temperature and solid density of the combustion models in Refs. [10,11] into a single reactionconvection-diffusion equation for the gas temperature. This simplification is obtained and follows from assuming a combustion process divided into two zones (see Fig. 1). In the preheating one, the solid density is almost constant and no vaporization occurs, whereas in the pyrolyzing region, the solid temperature reaches a constant value corresponding to the vaporization temperature $T_{v}$. The flame leading edge touches the surface at the location between both regions. Consistent with the previous assumption, we employ a flame model based 


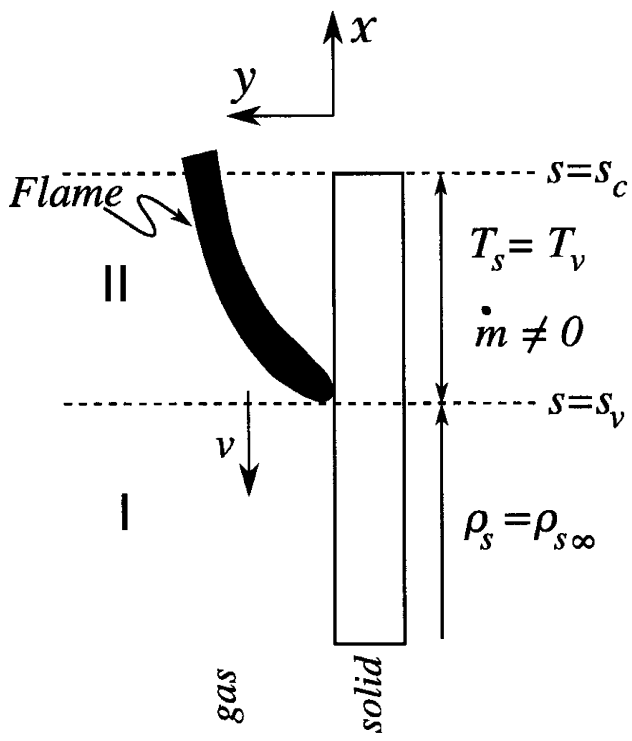

FIG. 1. Thin solid fuel combustion model employed in the present paper. The flame front velocity $v$ propagates downward (towards negative $x$ ).

on a simplified one-dimensional vertical structure where no oxidizer is found below the flame and no fuel above it [12].

The reaction-convection-diffusion equation is solved for both zones and the condition of matching the gas temperature and its derivative at the flame leading edge leads to the analytical expression for the flame front velocity. We also derive a simplified version of this analytical expression based on an order of magnitude analysis. Results from our analytical expressions are compared with experimental data found in the literature for a large variety of cases and clearly improve the predictions obtained after applying Eq. (1).

The structure of the paper is as follows. In Sec. II we describe the combustion model. In Sec. III we develop the method for deriving the analytical expressions for the flame front speed. In Sec. IV we carry out a comparison of the results obtained from our expressions with both experimental data and Eq. (1). Finally, we summarize the conclusions of the present work in Sec. V.

\section{COMBUSTION MODEL}

The combustion model follows those employed in Refs. [10,11] for performing numerical simulations of the downward burning process of a thin solid fuel. The governing equations for the solid phase assume a one-step reaction that follows a first-order Arrhenius law for the pyrolysis process, being

$$
\begin{aligned}
c_{s} \rho_{s} \frac{\partial T_{s}}{\partial t}= & \frac{\partial}{\partial x}\left(\lambda_{s} \frac{\partial T_{s}}{\partial x}\right)-\frac{\partial J_{s y}}{\partial y} \\
& -\frac{\partial \rho_{s}}{\partial t}\left[L+\left(c_{s}-c_{g}\right)\left(T_{s}-T_{\infty}\right)\right], \\
\frac{\partial \rho_{s}}{\partial t}= & -A \rho_{s}\left(e^{-E_{s} / R T_{s}}-e^{-E_{s} / R T_{\infty}}\right),
\end{aligned}
$$

where $T_{s}$ is the solid temperature, $t$ is time, $x$ is the coordinate parallel to the solid surface, and $y$ is positive when pointing toward the gas phase. We assume that the flame propagates toward negative $x$ (see Fig. 1). In Eqs. (2) and (3), $J_{s y}$ is the solid phase heat flux in the $y$ direction, $\lambda_{s}$ is the solid conductivity, $c_{g}$ is the gas specific heat, $L$ is the latent heat of vaporization, $R$ is the universal gas constant, and $A$ and $E_{s}$ are the preexponential factor and the activation energy for the solid phase pyrolysis reaction, respectively.

Equations for the gas phase are $[10,11]$

$$
\begin{gathered}
c_{g} \rho_{g} \frac{\partial T}{\partial t}+c_{g} \rho_{g} u_{x} \frac{\partial T}{\partial x}+c_{g} \rho_{g} u_{y} \frac{\partial T}{\partial y} \\
=\frac{\partial}{\partial x}\left(\lambda_{g} \frac{\partial T}{\partial x}\right)-\frac{\partial J_{g y}}{\partial y}+q \omega, \\
\rho_{g} \frac{\partial Y_{O}}{\partial t}+\rho_{g} u_{x} \frac{\partial Y_{O}}{\partial x}+\rho_{g} u_{y} \frac{\partial Y_{O}}{\partial y} \\
=\frac{\partial}{\partial x}\left(\rho_{g} D_{O} \frac{\partial Y_{O}}{\partial x}\right)+\frac{\partial}{\partial y}\left(\rho_{g} D_{O} \frac{\partial Y_{O}}{\partial y}\right)-f \omega, \\
\rho_{g} \frac{\partial Y_{F}}{\partial t}+\rho_{g} u_{x} \frac{\partial Y_{F}}{\partial x}+\rho_{g} u_{y} \frac{\partial Y_{F}}{\partial y} \\
=\frac{\partial}{\partial x}\left(\rho_{g} D_{F} \frac{\partial Y_{F}}{\partial x}\right)+\frac{\partial}{\partial y}\left(\rho_{g} D_{F} \frac{\partial Y_{F}}{\partial y}\right)-\omega,
\end{gathered}
$$

where $T$ is the gas temperature, $Y_{O}$ is the oxygen mass fraction, $Y_{F}$ is the fuel mass fraction, $\rho_{g}$ is the gas density, $D_{O}$ is the oxygen mass diffusivity, $D_{F}$ is the fuel mass diffusivity, $u$ is the background flow velocity, $q$ is the heat of combustion per unit mass of fuel, $f$ is the stoichiometric oxidizer to fuel mass ratio, $J_{g y}$ is the gas phase heat flux in the $y$ direction, and $\omega$ is the gas phase reaction rate

$$
\omega=B \rho_{g}^{2} Y_{O} Y_{F}\left(e^{-E_{g} / R T}-e^{-E_{g} / R T_{\infty}}\right),
$$

where, following Ref. [4], the last term within the parentheses has been included in order to assure $\partial T / \partial t=0$ at room conditions $\left(T=T_{\infty}\right)$ in a quiescent environment. In Eq. (7), $B$ is the preexponential factor and $E_{g}$ is the activation energy of a one-step reaction that follows a second-order Arrhenius law for the gas phase combustion.

Let us now reduce the number of partial differential equations (PDEs) in the gas phase by expressing both oxygen and fuel mass fractions in terms of the gas temperature [i.e., $Y_{O}=Y_{O}(T)$ and $\left.Y_{F}=Y_{F}(T)\right]$ that substituted into Eq. (7) will allow us to express Eq. (4) as a function of $T$ and $J_{g y}$ only.

For doing so, we assume a steady one-dimensional vertical flame model similar to that analyzed in Ref. [12] for the propagation of downward flame fronts in thin solid fuels with background flow at rest (i.e., $u_{x}=0$ ) and constant transport coefficients, gas density, and mass flux of volatiles $\dot{m}=\rho_{g} u_{y}$. Then, Eqs. (4)-(6) become

$$
\begin{gathered}
c_{g} \dot{m} \frac{d T}{d y}=\lambda_{g} \frac{d^{2} T}{d y^{2}}+q \omega, \\
\dot{m} \frac{d Y_{O}}{d y}=\rho_{g} D_{O} \frac{d^{2} Y_{O}}{d y^{2}}-f \omega, \\
\dot{m} \frac{d Y_{F}}{d y}=\rho_{g} D_{F} \frac{d^{2} Y_{F}}{d y^{2}}-\omega,
\end{gathered}
$$

where the heat flux in the $y$ direction is $J_{g y}=-\lambda_{g} d T / d y$. 
The time derivative terms in Eqs. (4)-(6) may be expressed in terms of the $s=x+v t$ coordinate attached to the flame front, where $v$ is the (positive) flame front speed. For simplicity, these terms plus the heat and mass diffusivity ones in the $s$ direction have been ignored in the vertical flame model (8)-(10). This strong assumption has been proven to yield reasonable spread rates in the study of downward flame fronts in thin solid fuels based on the deRis analysis for nonunity Lewis numbers [12].

For the one-dimensional vertical flame model, the boundary conditions are [12]

$$
\begin{aligned}
Y_{O o} & =0, \\
\left.\rho_{g} D_{F} \frac{d Y_{F}}{d y}\right|_{y=0} & =\dot{m}\left(Y_{F o}-1\right), \\
\left.\frac{d T}{d y}\right|_{y=0} & =\text { constant },
\end{aligned}
$$

at $y=0$ (solid surface), with $Y_{O o}=Y_{O}(y=0)$ and $Y_{F o}=$ $Y_{F}(y=0)$, and

$$
\begin{gathered}
Y_{O t}=Y_{O \infty}, \\
Y_{F t}=0, \\
T_{t}=T_{\infty},
\end{gathered}
$$

at $y=y_{t}$, where $Y_{O t}=Y_{O}\left(y=y_{t}\right), Y_{F t}=Y_{F}\left(y=y_{t}\right)$, and $T_{t}=T\left(y=y_{t}\right)$, with $y_{t}$ a distance far enough from the flame that both oxygen mass fraction and temperature are equal to the room values $Y_{O \infty}$ and $T_{\infty}$, respectively.

Note that, consistent with the assumption of a flame leading edge touching the surface with a vaporizing solid behind it, $Y_{O}=0$ for $y<y_{f}$ and $Y_{F}=0$ for $y>y_{f}, y_{f}$ being the flame height where $T\left(y=y_{f}\right)=T_{f}$. For simplicity we assume here a constant value for $y_{f}$, although in the real combustion process it is, indeed, a function of $s$ (see, e.g., [13]). Thus, the solutions of Eqs. (8)-(10) that satisfy the above boundary conditions are

$$
T= \begin{cases}T_{f}-\left.\frac{\lambda_{g}}{\dot{m} c_{g}}\left(e^{\dot{m} c_{g} y_{f} / \lambda_{g}}-e^{\dot{m} c_{g} y / \lambda_{g}}\right) \frac{d T}{d y}\right|_{y=0} & \text { for } 0 \leqslant y<y_{f} \\ T_{f}-\left(T_{f}-T_{\infty}\right) \frac{\left(e^{\dot{m} c_{g} y / \lambda_{g}}-e^{\dot{m} c_{g} y_{f} / \lambda_{g}}\right)}{\left(e^{\dot{m} c_{g} y_{t} / \lambda_{g}}-e^{\dot{m} c_{g} y_{f} / \lambda_{g}}\right)} & \text { for } y_{f}<y \leqslant y_{t},\end{cases}
$$

$$
\begin{aligned}
& Y_{O}= \begin{cases}0 & \text { for } 0 \leqslant y<y_{f} \\
Y_{O \infty} \frac{\left(e^{\dot{m} c_{g} y / \lambda g}-e^{\dot{m} c_{g} y_{f} / \lambda g}\right)}{\left(e^{\dot{m} c_{g} y_{t} / \lambda g}-e^{\dot{m} c_{g} y_{f} / \lambda g}\right)} & \text { for } y_{f}<y \leqslant y_{t},\end{cases} \\
& Y_{F}= \begin{cases}1-e^{\dot{m} c_{g}\left(y-y_{f}\right) / \lambda_{g}} & \text { for } 0 \leqslant y<y_{f} \\
0 & \text { for } y_{f}<y \leqslant y_{t},\end{cases}
\end{aligned}
$$

where, for simplicity, we have assumed a unit Lewis number Le [i.e., $D_{O}=D_{F}=\alpha_{g}$, where $\alpha_{g}=\lambda_{g} /\left(\rho_{g} c_{g}\right)$ is the gas mixture heat diffusivity].

From Eqs. (17)-(19), both oxygen and fuel mass fractions may be expressed in terms of the dimensionless gas temperature

$$
\theta=\frac{\left(T-T_{\infty}\right)}{\left(T_{f}-T_{\infty}\right)}
$$

being

$$
\begin{gathered}
Y_{O}=Y_{O \infty}(1-\theta) \quad \text { for } y>y_{f}, \\
Y_{F}=c_{g}\left(T_{f}-T_{\infty}\right) \frac{\dot{m}}{\left.\lambda_{g} \frac{d T}{d y}\right|_{y=0}} \frac{\ln \left(1+B_{c}\right)}{B_{c}}(1-\theta) \quad \text { for } y<y_{f},
\end{gathered}
$$

since $\dot{m}\left(1-Y_{F o}\right)=-\rho_{g} D_{F} d Y_{F} /\left.d y\right|_{y=0}=\dot{m} \ln \left(1+B_{c}\right) / B_{c}$ [7], where $B_{c}$ follows:

$$
B_{c}=\frac{Y_{O \infty} q}{L f}-\frac{c_{g}\left(T_{v}-T_{\infty}\right)}{L},
$$

being the adiabatic mass-transfer number first introduced by Spalding [14] and used by Emmons [15] and later by deRis [7] in order to linearize the boundary condition at the surface.

We point out that our combustion model shown in Fig. 1 assumes gas phase variables independent of the $y$ coordinate. This is equivalent to work with uniform values for the gas phase in the $y$ direction (except for the vertical heat flux $J_{g y}$ ) that may be also understood as averaged values for the $y$ coordinate in the gas phase. This approach for the gas phase is similar to the control volume analysis developed in Ref. [9] for the solid phase that finally led to Eq. (1). Note that we have obtained above the relationships $Y_{O}=Y_{O}(\theta)$ (21) and $Y_{F}=Y_{F}(\theta)$ (22) for a vertical thin sheet flame model that implicitly rejects the coexistence of oxidizer and fuel for $y \neq y_{f}$. However, when dealing with a single control volume in the $y$ dimension and, hence, with $\theta, Y_{O}$ and $Y_{F}$ uniform values for the gas phase in the $y$ direction, nonzero values of both oxygen and fuel mass fractions in region II of Fig. 1 are expected. In what follows we assume that the functions $Y_{O}=Y_{O}(\theta)$ (21) and $Y_{F}=Y_{F}(\theta)$ (22) reasonably explain the behavior of $Y_{O}$ and $Y_{F}$ in region II of Fig. 1 when working with $y$ uniform values and, for the sake of convenience, variables $Y_{O}, Y_{F}, \theta$, and $T$ will stand for such a $y$ independent values in our simplified combustion model. Indeed, the $Y_{O}=Y_{O}(\theta)$ and $Y_{F}=Y_{F}(\theta)$ 
dependences (21) and (22) reasonably follow those extracted from the contour plots shown in Refs. $[10,11,16]$ in the analysis of the downward spread rate of thin solid fuels by means of performing detailed numerical simulations.
Thus, the reaction-rate term $\omega$ for the gas phase in Eq. (7) is a function of the $\theta$ variable only, being

$$
\omega=\frac{B \rho_{g}^{2} Y_{O \infty} c_{g}\left(T_{f}-T_{\infty}\right)}{L} \frac{\ln \left(1+B_{c}\right)}{B_{c}}(1-\theta)^{2}\left(e^{-E_{g} R^{-1}\left[\theta\left(T_{f}-T_{\infty}\right)+T_{\infty}\right]^{-1}}-e^{-E_{g} R^{-1} T_{\infty}^{-1}}\right) \quad \text { for } s_{v} \leqslant s \leqslant s_{c}
$$

where we have assumed that all of the heat flux at the surface coming from the gas phase is used to vaporize the solid [i.e., $\dot{m} L=\lambda_{g} d T /\left.d y\right|_{y=0}$ in Eq. (22)]. Of course this assumption gives us a value of $\omega$ greater than the actual one so our method is expected to predict a spread rate of the flame front higher than that observed. In Eq. (24) $s_{v}$ is the position of the flame leading edge that coincides with the starting point of solid vaporization, whereas $s_{c}$ is the position of the complete burning of the solid.

Equation (24) substituted into Eq. (4) leads to a reactionconvection-diffusion equation as a function of $T$ only (or, equivalently, $\theta$ ) that is coupled to the solid-phase Eqs. (2) and (3) by means of the $y$-heat flux at the surface $J_{g y}$ and the mass flux of volatiles $\dot{m}$. These last two terms are expressed in terms of the simple behavior for the solid phase shown in Fig. 1. Indeed, this procedure is the opposite to that carried out in Ref. [9] for obtaining Eq. (1) since Bhatacharjee et al. used a simplified description of the gas phase as a boundary condition for the control volume of the solid phase.

Based on the results obtained from detailed numerical simulations for the burning rate of downward thin solid fuels shown in Refs. [10,11,16,17], the solid phase in these types of experiments may be divided into two main regions. Region I in Fig. 1 is ahead of the flame front and corresponds to the preheating zone $\left(s<s_{v}\right)$. In this zone the solid temperature increases due to the heat transfer from both gas and solid phases, although the last contribution is expected to become of primary importance for thick solid fuels only [18]. In region I the solid density does not vary since vaporization is not reached. On the other hand, region II is behind the flame and the solid is vaporizing $\left(s_{v} \leqslant s \leqslant s_{c}\right)$. For simplicity we assume a constant value for the vaporization temperature $T_{v}$ in region II, whereas the solid density decreases due to the release of volatiles and the formation of char. Note that we may add a third region such as $s_{c}<s$, irrelevant for our study since it corresponds to the burn out of the solid fuel with char releasing.

We parametrize the solid fuel density as a function of the distance from the flame leading edge position $s_{v}$ as

$$
\rho_{s}= \begin{cases}\rho_{s \infty}, & s<s_{v}, \\ \frac{\left(s_{c}-s\right)}{\left(s_{c}-s_{v}\right)} \rho_{s \infty}, & s_{v} \leqslant s \leqslant s_{c} \\ 0, & s_{c}<s\end{cases}
$$

where $s_{c}-s_{v}$ corresponds to the characteristic length of the vaporizing region and $\rho_{s}$ is the density of the virgin solid at room temperature. This linear behavior of $\rho_{s}$ in terms of $s$ reasonably reproduces the results obtained in Refs. [10,11,16,17] employing numerical simulations.

On the other hand, the mass flux of volatiles $\dot{m}$ follows from [17]

$$
\dot{m}=\int_{0}^{\delta_{s}} d y\left(-\frac{\partial \rho_{s}}{\partial t}\right) \simeq-\delta_{s} v \frac{d \rho_{s}}{d s},
$$

where the last approximation applies a uniform density of the solid in the $y$ direction in agreement with the thin solid fuel assumption so $\rho_{s}(x, y, t)=\rho_{s}(x, t)=\rho_{s}(s)$ with $s=x+v t$ and $\partial \rho_{s} / \partial t=v d \rho_{s} / d s$.

Substituting Eq. (25) into (26) we obtain

$$
\dot{m}= \begin{cases}0, & s<s_{v}, \\ a \delta_{s} v^{2} \frac{\rho_{s \infty}}{\alpha_{s}}, & s_{v} \leqslant s \leqslant s_{c}, \\ 0, & s_{c}<s,\end{cases}
$$

where the characteristic length of the vaporizing region $s_{c}-s_{v}$ corresponds to the thermal length for the solid phase $\alpha_{s} / v$ [19] [i.e., $\left(s_{c}-s_{v}\right) \simeq \alpha_{s} / v$ ]. Note that in Eq. (27) we have included the constant parameter $a$ with the aim of taking into account some of the errors we may encounter when adopting the approximation (26) and that for the characteristic length of the vaporizing region. The analysis of the results obtained from numerical simulations in Ref. [17] for $\delta_{s}=0.15 \times 10^{-3}, 0.3 \times 10^{-3}, 0.5 \times 10^{-3}$, and $0.7 \times 10^{-3}$ $\mathrm{m}$ produces a ratio $\left[\int \dot{m} d s /\left(s_{c}-s_{v}\right)\right] /\left(\delta_{s} v^{2} \rho_{s \infty} / \alpha_{s}\right)$ equal to $0.182,0.241,0.259$, and 0.236 , respectively, whose mean value $(=0.229)$ does not coincide with the expected $a=1$ value. Unless otherwise stated, we apply $a=0.229$ in what follows. Section IV includes a discussion on the implications of using $a=1$ in Eq. (27).

Finally, the $y$ heat flux from the gas phase into the solid phase follows the parametrization applied by Ref. [19], with an exponential decay of the heat flux ahead of the flame (region I) and a constant value behind it (region II),

$$
\left.J_{g y}\right|_{y=0}= \begin{cases}-J_{0} e^{\left(s-s_{v}\right) / l}, & s<s_{v}, \\ -J_{0}, & s_{v} \leqslant s \leqslant s_{c}, \\ 0, & s_{c}<s,\end{cases}
$$

where $J_{0}$ is a positive constant and $l$ is the characteristic thermal length for the decaying of the heat flux ahead of the flame. Unless otherwise stated, we assume here that $l=\alpha_{g} / U$ with $\alpha_{g}$ the gas mixture thermal diffusivity and $U\left(\approx 0.3 \mathrm{~m} \mathrm{~s}^{-1}\right)$ the induced flow velocity due to density variations near the flame when the background flow is 
initially (and at the boundaries) at rest [11]. Note that $\alpha_{g} / U$ corresponds to the characteristic thermal length in the gas phase [19]. In Sec. IV we also investigate the implications of using different values of $l$.

We point out that $J_{g_{0}}=\left.J_{g y}\right|_{y=0}$ is negative (i.e., heat goes toward the solid) since the gas phase temperature is assumed to be greater than the vaporization temperature in region II. No value of the heat flux from the gas to the solid is assumed for $s_{c}<s$ since char has been released.

The divergence of the vertical heat flux in the gas phase zone is approximated as $-\partial J_{g y} / \partial y \approx-\left(J_{g \delta y}-J_{g 0}\right) / \delta_{y}=J_{g_{0}} / \delta_{y}$, where $\delta_{y}$ is a characteristic thermal length for the gas phase such as $\left|J_{g \delta y}\right| \ll\left|J_{g 0}\right|$, where $J_{g \delta y}=\left.J_{g y}\right|_{y=\delta y}$. From Eq. (28) this leads to

$$
-\frac{\partial J_{g y}}{\partial y}= \begin{cases}-\frac{J_{0}}{\delta_{y}} e^{\left(s-s_{v}\right) / l}, & s<s_{v}, \\ -\frac{J_{0}}{\delta_{y}}, & s_{v} \leqslant s \leqslant s_{c}, \\ 0 . & s_{c}<s .\end{cases}
$$

\section{FLAME FRONT SPEED}

Let us now derive an analytical expression for the flame front speed from the combustion model described in the preceding section. For the sake of convenience we define the dimensionless variables $t^{\prime}, x^{\prime}, v^{\prime}$ and parameters $\gamma$ and $\beta$ as

$$
\begin{gathered}
\gamma=\frac{T_{f}-T_{\infty}}{T_{f}}, \quad \beta=\frac{\gamma E}{R T_{f}}, \\
t^{\prime}=t \frac{B \rho_{g} \gamma^{2}}{\beta}, \quad x^{\prime}=x\left(\frac{B \rho_{g} \gamma^{2}}{\alpha_{g} \beta}\right)^{1 / 2}, \\
v^{\prime}=v\left(\frac{\beta}{\alpha_{g} B \rho_{g} \gamma^{2}}\right)^{1 / 2} .
\end{gathered}
$$

\section{A. Region II}

We first analyze region II since it will allow us to introduce the maximum temperature that will be employed to renormalize the equations. For region II the substitution of Eqs. (30)-(32) into Eq. (4) leads to

$$
\begin{aligned}
v^{\prime} \frac{d \theta}{d s^{\prime}}= & \frac{d^{2} \theta}{d s^{\prime 2}}-\frac{\beta}{B \rho_{g} \gamma^{2}} \frac{1}{\rho_{g} c_{g}\left(T_{f}-T_{\infty}\right)} \frac{J_{0}}{\delta_{y}} \\
& -c_{g} \delta_{s} v^{2} \frac{\rho_{s \infty}}{\alpha_{s}} \frac{\beta}{B \rho_{g} \gamma^{2}} \frac{1}{\rho_{g} c_{g}\left(T_{f}-T_{\infty}\right)} \frac{J_{0}}{\lambda_{g}} \\
& +\frac{\beta}{B \rho_{g} \gamma^{2}} \frac{1}{\rho_{g} c_{g}\left(T_{f}-T_{\infty}\right)} q \omega,
\end{aligned}
$$

where $s^{\prime}$ is the dimensionless coordinate attached to the flame front that satisfies $s^{\prime}=x^{\prime}+v^{\prime} t^{\prime}$, with $v^{\prime}$ being the positive dimensionless flame front velocity (the front propagates downward in Fig. 1). Note that in Eq. (33), $\omega$ follows Eq. (24) and we have made use of the $\dot{m}=\rho_{g} u_{y}$ definition with $c_{g} \dot{m} \partial T / \partial y \approx c_{g} \delta_{s} a v^{2} \rho_{s \infty} J_{0} /\left(\alpha_{s} \lambda_{g}\right)$ since $\partial T / \partial y \approx J_{0} / \lambda_{g}$ in region II.
Let us define the parameters

$$
\begin{gathered}
F=\frac{\beta}{B \rho_{g} \gamma^{2}} \frac{1}{\rho_{g} c_{g}\left(T_{f}-T_{\infty}\right)} \frac{J_{0}}{\delta_{y}}, \\
G=c_{g} \delta_{s} \frac{\rho_{s}}{\alpha_{s}} \frac{\delta_{y}}{\lambda_{g}} \frac{\alpha_{g} B \rho_{g} \gamma^{2}}{\beta}, \\
H=\frac{\beta}{B \rho_{g} \gamma^{2}} \frac{q \omega}{\rho_{g} c_{g}\left(T_{f}-T_{\infty}\right)},
\end{gathered}
$$

that substituted into Eq. (33) yield

$$
v^{\prime} \frac{d \theta}{d s^{\prime}}=\frac{d^{2} \theta}{d s^{\prime 2}}-F\left(1+G v^{\prime 2}\right)+H,
$$

which is the one-dimensional reaction-convection-diffusion equation for the single variable $\theta$ that will allow us to determine the flame front speed.

We assume that the wave front arisen from Eq. (37) reaches extremum values of the dimensionless temperature $\theta$ for those points where $d \theta / d s^{\prime}=0$. Of course, this condition cannot be satisfied for $\theta \rightarrow 0$ since the minimum temperature value that can be attained in region II is $\theta_{v}=\left(T_{v}-T_{\infty}\right) /\left(T_{f}-T_{\infty}\right)$. In the limit $\theta \rightarrow 1, H=0$ but $F \neq 0$ in Eq. (37), which implies $d \theta /\left.d s^{\prime}\right|_{\theta \rightarrow 1} \neq 0$. Therefore, we expect that $\theta$ reaches a maximum temperature $\theta_{\max }$ that satisfies

$$
H\left(\theta_{\max }\right)=F\left(1+v^{\prime 2} G\right)
$$

and, from Eq. (37), $d \theta /\left.d s^{\prime}\right|_{\theta \rightarrow \theta_{\max }}=0$. Since $F, G$, and $v^{\prime}$ are positive values and $H \geqslant 0$ for $0 \leqslant \theta \leqslant 1$ with $H \rightarrow 0$ for both $\theta=0$ and $\theta=1$ limits, we will obtain $\theta_{\max }<1$. This condition implicitly assumes $T_{\max }<T_{f}$ in the gas phase, which may be explained from the essentially $y$-averaged feature of the variables in our simple model. Indeed, the actual temperature distribution in the $y$ direction in region II of Fig. 1 does not correspond to a uniform $T_{f}$ value since the flame occupies a thin slab at $y=y_{f}$. Therefore, we expect to use $T<T_{f}$ when working with a single temperature for the whole $y$ dimension. We point out that Eq. (38) involves the unknown dimensionless flame front speed $v^{\prime}$, so its solution requires an iterative process that we will explain below. In addition, Eq. (38) may have more than one root. In this case, the maximum value is $\theta_{\max }(<1)$.

Let us now work with the new dimensionless temperature

$$
\theta^{\prime}=\frac{\theta}{\theta_{\max }},
$$

so region I and II ranges from $0<\theta^{\prime}<1$ with $d \theta^{\prime} /\left.d s^{\prime}\right|_{\theta^{\prime} \rightarrow 1}=$ 0 .

By substituting Eq. (39) into Eq. (37) we obtain

$$
\frac{d^{2} \theta^{\prime}}{d s^{\prime 2}}-v^{\prime} \frac{d \theta^{\prime}}{d s^{\prime}}-K\left(1+v^{\prime 2} G\right)+I=0,
$$

where $K=F / \theta_{\max }$ and $I=H / \theta_{\max }$.

As it has been extensively applied in other works dealing with variational techniques for determining the wave front velocity [20], we now work in the phase space $p=d \theta^{\prime} / d s^{\prime}$, so Eq. (40) reads

$$
p \frac{d p}{d \theta^{\prime}}-v^{\prime} p-K\left(1+v^{\prime 2} G\right)+I=0 .
$$


Let us integrate Eq. (41) from $\theta^{\prime}\left(s^{\prime}=s_{v}^{\prime}\right)=\theta_{v}^{\prime}$ to $\theta^{\prime}=1$ (i.e., for all region II), giving

$$
\begin{aligned}
& \frac{\left(p_{1}^{2}-p_{v}^{2}\right)}{2}-v^{\prime} \frac{\left(p_{1}+p_{v}\right)}{2}\left(1-\theta_{v}^{\prime}\right) \\
& \quad-K\left(1+v^{\prime 2} G\right)\left(1-\theta_{v}^{\prime}\right)+I^{\prime}=0,
\end{aligned}
$$

where $p_{1}=p\left(\theta^{\prime}=1\right), p_{v}=p\left(\theta^{\prime}=\theta_{v}^{\prime}\right), I^{\prime}=\int_{\theta_{v}^{\prime}}^{1} I d \theta^{\prime}$, and the integration of the term $\int_{\theta_{v}^{\prime}}^{1} p d \theta^{\prime}$ has been approximated by using a mean value of $p$ for the $\theta_{v}^{\prime} \leqslant \theta^{\prime} \leqslant 1$ interval. This leads to $\int_{\theta_{u}^{\prime}}^{1} p d \theta^{\prime}=\left(1-\theta_{v}^{\prime}\right)\left(p_{1}+p_{v}\right) / 2$. Note that this type of approach has been successfully applied in the derivation of the flame front speed in gaseous fuels [21].

Since $\theta_{\max }$ is such that $p_{1}=p\left(\theta^{\prime}=1\right)=d \theta^{\prime} /\left.d s^{\prime}\right|_{\theta^{\prime} \rightarrow 1}=$ 0 , the solution for $p_{v}$ in Eq. (42) is

$$
p_{v \mathrm{II}}=-\frac{v^{\prime}}{2}\left(1-\theta_{v}^{\prime}\right)+\frac{1}{2} \sqrt{v^{\prime 2}\left(1-\theta_{v}^{\prime}\right)^{2}-4 K\left(1+v^{\prime 2} G\right) 2\left(1-\theta_{v}^{\prime}\right)+8 I^{\prime}},
$$

where the subscript II indicates that $p_{v}$ has been obtained from solving the equations in region II.

\section{B. Region I}

Equation (4) for region I in dimensionless variables (30)(32) reads

$$
\begin{aligned}
c_{g} \rho_{g}\left(T_{f}-T_{\infty}\right) \frac{B \rho_{g} \gamma^{2}}{\beta} \frac{\partial \theta}{\partial t^{\prime}} \\
\quad=\left(T_{f}-T_{\infty}\right) \frac{B \rho_{g} \gamma^{2}}{\alpha_{g} \beta} \frac{\partial}{\partial x^{\prime}}\left(\lambda_{g} \frac{\partial \theta}{\partial x^{\prime}}\right)-\frac{\partial J_{g y}}{\partial y},
\end{aligned}
$$

that in terms of $\theta^{\prime}$ and $s^{\prime}$, with Eq. (29) for expressing the heat flux divergence, is

$$
\frac{d^{2} \theta^{\prime}}{d s^{\prime 2}}-v^{\prime} \frac{d \theta^{\prime}}{d s^{\prime}}=K e^{\left(s^{\prime}-s_{v}^{\prime}\right) / l^{\prime}}
$$

where $l^{\prime}=l \sqrt{B \rho_{g} \gamma^{2} /\left(\alpha_{g} \beta\right)}$ is the dimensionless characteristic thermal length of the gas phase.
The solution of Eq. (45) with the condition $\theta^{\prime} \rightarrow 0$ for $s^{\prime} \rightarrow-\infty$ leads to

$$
\theta^{\prime}=c_{1} e^{v^{\prime} s^{\prime}}+\frac{K e^{\left(s^{\prime}-s_{v}^{\prime}\right) / l^{\prime}} l^{\prime}}{\left(-v^{\prime}+1 / l^{\prime}\right)},
$$

where the constant of integration $c_{1}$ is chosen such as $\theta^{\prime}=\theta_{v}^{\prime}$ at $s^{\prime}=s_{v}^{\prime}$.

From Eq. (46), the phase space variable in region I $p_{\mathrm{I}}=$ $d \theta^{\prime} / d s^{\prime}$ reads

$$
p_{\mathrm{I}}=\theta^{\prime} v^{\prime}+K l^{\prime} e^{\left(s^{\prime}-s_{v}^{\prime}\right) / l^{\prime}} .
$$

\section{Matching conditions at the flame leading edge}

The flame spread rate is obtained after applying matching conditions for $\theta^{\prime}$ and $p$ for both regions at the flame leading edge. This technique has been also employed for deriving analytical expressions for the burning velocity of flame fronts in gaseous fuels [21,22] and in more general systems modeled by reaction-convection-diffusion equations [23]. At the flame leading edge, $\theta^{\prime}=\theta_{v}^{\prime}$ and

$$
p_{v \mathrm{I}}=p_{v \mathrm{II}}
$$

so from Eqs. (43) and (47) at $\theta^{\prime}=\theta_{v}^{\prime}$ we obtain a second order algebraic equation on $v^{\prime}$, whose positive solution is

$$
v^{\prime}=-\frac{1}{2} \frac{\left(1+\theta_{v}^{\prime}\right) K l^{\prime}}{\left[\theta_{v}^{\prime}+2 K G\left(1-\theta_{v}^{\prime}\right)\right]}+\sqrt{\left[\frac{1}{2} \frac{\left(1+\theta_{v}^{\prime}\right) K l^{\prime}}{\left[\theta_{v}^{\prime}+2 K G\left(1-\theta_{v}^{\prime}\right)\right]}\right]^{2}+\frac{\left[2 I^{\prime}-2 K\left(1-\theta_{v}^{\prime}\right)-K^{2} l^{\prime 2}\right]}{\left[\theta_{v}^{\prime}+2 K G\left(1-\theta_{v}^{\prime}\right)\right]}} .
$$

In Eq. (49) $K=F / \theta_{\max }$ is a function of the absolute value of the heat flux at the surface $J_{0}$ in region II. Consistent with the assumptions adopted for solving the one-dimensional vertical flame model, we accept that the $y$-heat flux is entirely devoted to vaporize the solid. This implies that $\dot{m} L=\lambda_{g} \partial T /\left.\partial y\right|_{y=0}$ $=J_{0}$ and, from (27), (34), and the text below (40), we have

$$
K=\frac{\delta_{s}}{\theta_{\max }} \frac{a L \rho_{s \infty}}{\rho_{g} c_{g}\left(T_{f}-T_{\infty}\right) \delta_{y}} \frac{\alpha_{g}}{\alpha_{s}} v^{\prime 2} .
$$

Note that Eq. (50) is a function of $v^{\prime}$ that substituted into Eq. (49) leads to an implicit equation for $v^{\prime}$. The iterative procedure implemented for obtaining the solution is as follows. For a fixed value of $\theta_{v}^{\prime}$ (the temperature at the flame leading edge $s_{v}^{\prime}$ ), we find the roots of Eq. (38) by taking into consideration that, for any trial value of $\theta_{\max }$, the velocity $v^{\prime}$ satisfies Eq. (49) within a $10^{-5}$ relative error.

Finally, we have realized that common values of the parameters for the downward burning of thin cellulosic-type 
TABLE I. Values of the main gas and solid phase parameters. $M$ is the molar mass of the gas mixture. For other values see the text.

\begin{tabular}{|c|c|c|c|c|}
\hline Name & Symbol & Ref. [11] & Ref. [17] & Unit \\
\hline Preexponential factor & $B$ & $10^{6} / M$ & $9.02 \times 10^{7} / M$ & $\mathrm{~m}^{3} \mathrm{~kg}^{-1} \mathrm{~s}^{-1}$ \\
\hline Activation energy & $E_{g}$ & 87200 & 112860 & $\mathrm{~J} \mathrm{~kg}^{-1} \mathrm{~mol}^{-1}$ \\
\hline Heat of combustion per unit mass of fuel & $q$ & $1.674 \times 10^{7}$ & $1.672 \times 10^{7}$ & $\mathrm{~J} \mathrm{~kg}^{-1}$ \\
\hline Solid conductivity & $\lambda_{s}$ & 0.1255 & 0.105 & $\mathrm{~W} \mathrm{~m}{ }^{-1} \mathrm{~K}^{-1}$ \\
\hline Solid specific heat & $c_{s}$ & 1260 & 1460 & $\mathrm{~J} \mathrm{~K}^{-1} \mathrm{~kg}^{-1}$ \\
\hline Density of solid virgin material & $\rho_{s \infty}$ & 750 & 650 & $\mathrm{~kg} \mathrm{~m}^{-3}$ \\
\hline Vaporization temperature & $T_{v}$ & 618 & 700 & $\mathrm{~K}$ \\
\hline Latent heat of vaporization & $L$ & $753 \times 10^{3}$ & $418 \times 10^{3}$ & $\mathrm{~J} \mathrm{~kg}^{-1}$ \\
\hline
\end{tabular}

fuels imply $\theta_{v}^{\prime} \ll K G, l^{\prime} \ll G, K \ll I^{\prime}$, and $K^{2} l^{\prime 2} \ll I^{\prime}$ with $\theta_{v}^{\prime} \ll 1$. Under these conditions and by defining $K^{\prime}=K / v^{\prime 2}$, Eq. (49) reduces to

$$
v_{a}^{\prime}=\left(\frac{I^{\prime}}{K^{\prime} G}\right)^{1 / 4}
$$

which is the approximate explicit expression for the dimensionless flame front speed $v^{\prime}$.

\section{RESULTS AND COMPARISON TO EXPERIMENTS}

We investigate the validity of Eqs. (49) and (51) by comparing with available experimental data for thin cellulosictype fuels in cases that differ in oxygen concentration, fuel thickness, and inert gas type.

Values of the basic parameters for both gas and solid phases required by our analytical expressions are not provided in the experimental works cited here and, therefore, we have extracted them from previous studies that developed numerical simulations of the downward flame propagation of thin cellulosic-type fuels. We have noted that values of the solid phase parameters found in the literature do not differ in excess since they can be obtained experimentally by simple techniques. This is not the case for the kinetic constants of the gas phase chemical reaction since, indeed, the actual combustion reaction is more complex than a single-step second order Arrhenius type one. Gas chromatography analyses have revealed that pyrolysis of cellulose releases several volatiles that certainly react in a multistep way [24].

Nevertheless, the single-step second order gas phase reaction has been widely used in the numerical simulation of combustion of cellulosic-type fuels, where the kinetic constants have been either adopted from data of well-known fuels such as methane [25] or chosen to produce flame front speeds consistent with experimental data [10]. These procedures for determining the gas phase kinetic values do not necessarily produce identical results as it may be seen in Table I that summarises the values of the main gas and solid phase parameters employed in Refs. [11,17] for simulating the downward flame propagation of thin cellulosic-type fuels. We point out that other authors assume values of the kinetic constants that are within the range of values shown in Table I [26].

Here we have performed our analysis by taking both sets of values in Table I into consideration. Therefore we expect that the analytical results corresponding to the (unknown) values of the kinetic constants that better represent the actual gas phase reaction in terms of a single-step second order one will lie within the range of values obtained when applying the two sets of parameters shown in Table I.

In agreement with [27], transport coefficients for the gas phase as well as the gas density value have been evaluated at the reference temperature $\left(T_{f}+T_{\infty}\right) / 2$, where $T_{f}$ is the flame temperature that here follows the adiabatic flame expression [12]

$$
T_{f}=T_{\infty}+\frac{q}{c_{g}} \frac{Y_{O \infty}}{f}\left[1-\frac{B_{c} L}{q \ln \left(1+B_{c}\right)}\right]
$$

Thermal conductivities and specific heats of gases as a function of temperature follow from Ref. [28]. Gas mixture properties apply the classical ideal gas formulas [29]. All results assume a Lewis number equal to 1 and a dimensionless temperature $\theta_{v}=\left(T_{v}-T_{\infty}\right) /\left(T_{f}-T_{\infty}\right)$, with $\theta_{v}^{\prime}=\theta_{v} / \theta_{\max }$.

Analytical and experimental values of the flame front velocity as a function of the initial room oxygen molar concentration are shown in Fig. 2(a) for an atmosphere with Ar as a diluent and a cellulosic-type solid with $\delta_{s} \rho_{s \infty}=0.040 \mathrm{~kg} \mathrm{~m}^{-2}$, and in Fig. 2(b) for an atmosphere with $\mathrm{N}_{2}$ as a diluent and a cellulosic-type solid with $\delta_{s} \rho_{s \infty}=0.0175 \mathrm{~kg} \mathrm{~m}^{-2}$. Note that these $\delta_{s} \rho_{s}$ values agree with the thin solid fuel assumption (solid half-thickness $\delta_{s}$ lower than the characteristic solid thermal length) that from Ref. [17] is expected to be valid for $\delta_{s} \rho_{s \infty}<1.43 \mathrm{~kg} \mathrm{~m}^{-2}$.

For a fixed value of oxygen molar fraction at room conditions $\left(X_{O 2 \infty}=0.3\right)$, the flame front speed as a function of $\delta_{s} \rho_{s \infty}$ is shown in Fig. 3(a) with Ar as a diluent and in Fig. 3(b) with $\mathrm{N}_{2}$ as a diluent. We note that from Figs. 2 and 3 and in comparison with Eq. (1), Eq. (49) better explains the behavior of experimental data extracted from different authors [12,30-32].

For clarity, Figs. 2 and 3 do not include results obtained from the approximated expression Eq. (51). This last formula is compared with Eq. (49) in Fig. 4 for those cases shown in Fig. 2. Figure 4 reveals that Eq. (51) provides a very good estimate of Eq. (49) for thin solid fuels, mainly for values of the oxygen molar fraction $X_{O 2 \infty}>0.2$. For lower values, the decrease in $I^{\prime}$ may be so large that some of the conditions stated above Eq. (51) may not be satisfied. On the other hand, differences between Eqs. (51) and (49) for those cases shown in Fig. 3 are less than $3.5 \%$.

Let us now analyze the sensitivity of the analytical expressions to changes in the main physical parameters of 


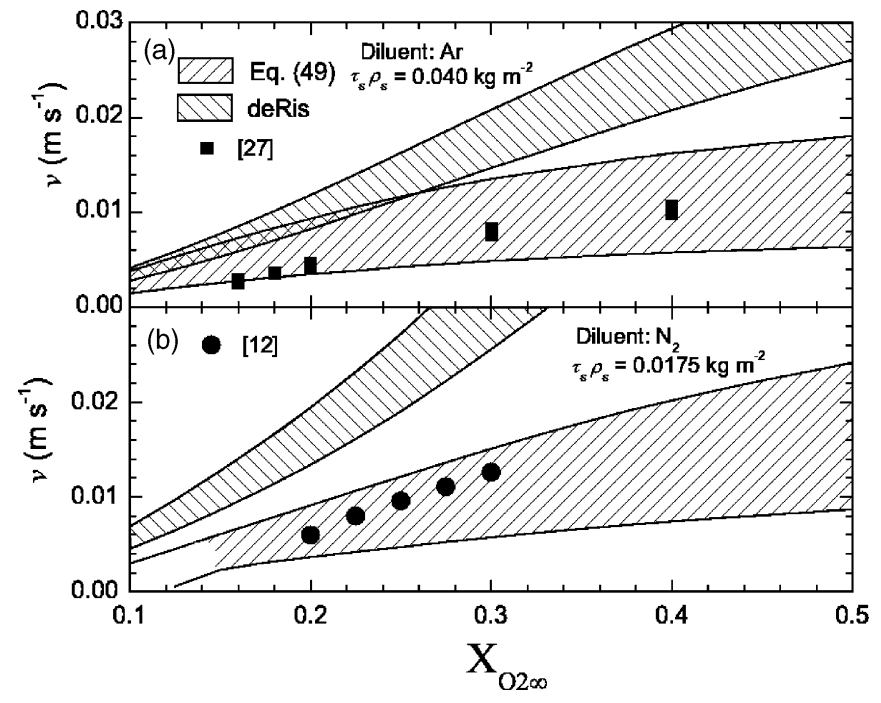

FIG. 2. (a) and (b) Flame front speed as a function of the oxygen molar fraction at room conditions for the downward burning of a cellulosic-type solid with $\delta_{s} \rho_{s \infty}=0.040 \mathrm{~kg} \mathrm{~m}^{-2}$ and $\mathrm{Ar}$ as a diluent (a), and with $\delta_{s} \rho_{s \infty}=0.0175 \mathrm{~kg} \mathrm{~m}^{-2}$ and $\mathrm{N}_{2}$ as a diluent (b). Symbols correspond to experimental data, whereas deRis' hatched area is the classical analytical expression Eq. (1).

both gas and solid phases. For the sake of simplicity we work with Eq. (51) instead of Eq. (49) that from Eqs. (32), (35), and (50) reads

$$
v_{a}=\left(\frac{\lambda_{s}}{c_{s} \delta_{s} \rho_{s}^{2}}\right)^{1 / 2}\left(\frac{\lambda_{g}}{c_{g}} \frac{q}{L}\right)^{1 / 4}\left(\frac{1}{\theta_{\max }} \int_{\theta_{v}^{\prime}}^{1} \omega d \theta^{\prime}\right)^{1 / 4},
$$

where the reaction-rate $\omega$ is defined in Eq. (24).

Both Eqs. (1) and (53) depend on the main gas phase properties $\lambda_{g}$ and $c_{g}$, solid phase ones $c_{s}, \delta_{s}, \rho_{s}$, combustion heat release per unit mass of fuel $q$, latent heat of vaporization $L$, mass fraction of oxygen at room conditions $Y_{O \infty}$, vaporization

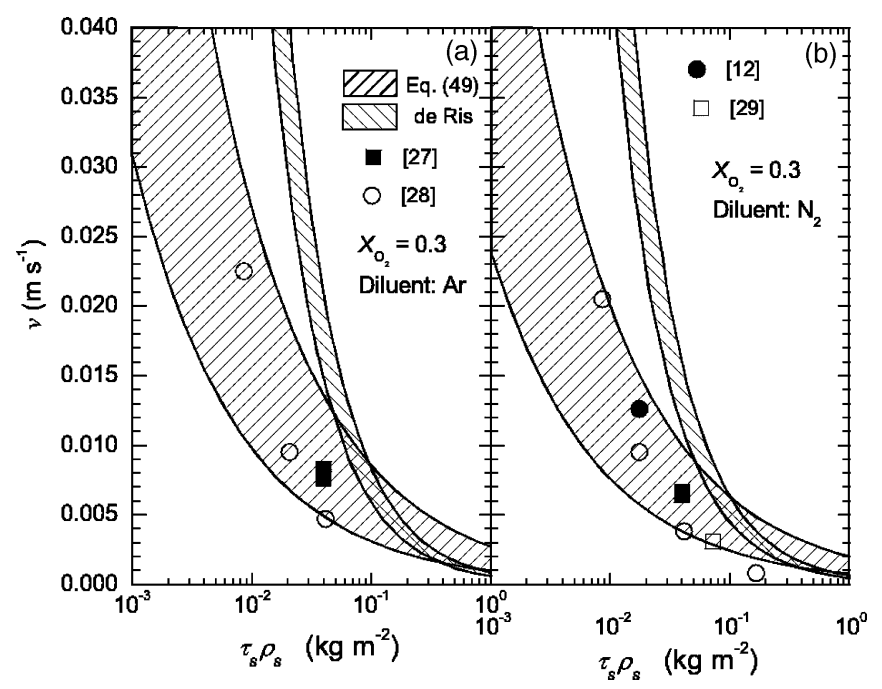

FIG. 3. (a) and (b) Flame front speed as a function of $\delta_{s} \rho_{s \infty}$ for the downward burning of a cellulosic-type solid with oxygen molar fraction at room conditions $X_{O 2 \infty}=0.3$ and $\mathrm{Ar}$ as a diluent (a) and $\mathrm{N}_{2}$ as a diluent (b). Symbols correspond to experimental data whereas deRis' hatched area is the classical analytical expression Eq. (1).

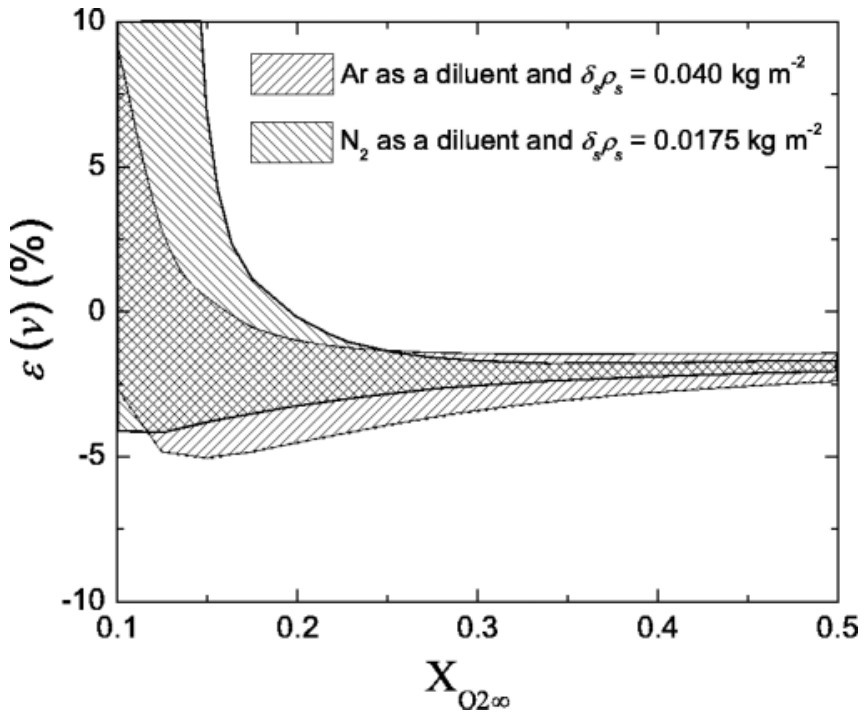

FIG. 4. Differences of the approximate expression Eq. (51) with respect to Eq. (49) $\varepsilon(v)=100\left(v_{a}^{\prime}-v^{\prime}\right) / v^{\prime}$ for those cases analyzed in Figs. 2(a) and 2(b).

temperature $T_{v}$, flame temperature $T_{f}$, and room temperature $T_{\infty}$. Note that in Eq. (53) the dependence of $v_{a}$ on $Y_{O \infty}$, $T_{f}$, and $T_{\infty}$ is through $\omega$. In Eq. (1) the dependence of $v$ on $c_{g}, q, L, Y_{O \infty}$ is through $T_{f}$. For both (1) and (53) expressions, an increase in $\lambda_{g}, q, Y_{O \infty}, T_{\infty}$, and $T_{f}$ leads to an increase in the flame front velocity (as a priori expected), whereas an increase in $c_{s}, \delta_{s}, \rho_{s}, c_{g}, L$, and $T_{v}$ leads to a decrease in the flame front velocity (as a priori also expected). However, the sensitivity of the solution to changes in the parameters may substantially differ for both (1) and (53) analytical expressions. Thus, for example, Eq. (1) predicts $v \propto \delta_{s}^{-1}$, whereas Eq. (53) proposes $v_{a} \propto \delta_{s}^{-1 / 2}$ as we have shown in Fig. 3 .

It is very important to stress, however, that in contrast with the classical deRis Eq. (1), our analytical expressions for the flame front speed Eqs. (49) and (53) depend on the preexponential factor $B$ and activation energy $E_{g}$ of the gas phase combustion reaction and on the solid conductivity $\lambda_{s}$. Thus, Eqs. (49) and (53) become a function of some relevant physical parameters that are not taken into account in Eq. (1). Indeed, the influence of the heat transfer through the solid in the flame front speed of thin solid fuels has been a controversial topic. This effect has been included in complex numerical simulations (e.g., [17]) while being ignored when deriving Eq. (1) from a simple control volume analysis [9]. Here, Eq. (53) may shed light into the implications of this parameter on the flame spread rate.

On the other hand, the discrepancies of the values for the kinetic constants $B$ and $E_{g}$ are the main causes of the large zones shown in Figs. 2 and 3 for our analytical results. Indeed, the validity of using a single-step second order Arrhenius type reaction as an approximation for the actual gas phase behavior has been analyzed in Ref. [33], where multistep chemical kinetic models for the gas phase reactions are implemented. Wolverton et al. [33] found that far from the extinction conditions, multistep kinetic models yield similar spread rates than those obtained from the single-step one (differences lower than $10 \%$ ), although they clearly improve the predictions for 
the flame structure. Near the extinction condition, however, gas phase kinetics become the main mechanism for controlling the heat release. In this case, single-step models predict flame front speeds at least $60 \%$ greater than those obtained from the multistep ones. Indeed, the single-step approximation for the gas phase used may be the reason why our analytical expressions fail to predict an extinction point (see, e.g., Ref. [33]). From the above, the simplification of the gas phase reaction in terms of an overall single-step second order Arrhenius type one appears reasonable when working far from the extinction.

Finally, we have performed several studies varying some of the key parameters involved in the assumptions done for deriving Eq. (49). For example, changes in the characteristic thermal length $l$ lead to small changes in the flame front speed in Eq. (49) (decrease smaller than 3\% when doubling $l$ for the cases shown in Fig. 2). We note that this parameter is not taken into consideration in the approximated expression Eq. (53) that, similarly to Eq. (1), is independent of the induced flow velocity $U$. For large values of $U$, however, the assumption of a background flow at rest may not be valid and the proportionality $\dot{m} \propto \delta_{s} v^{2} \rho_{s \infty} / \alpha_{s}$ used in Eq. (27) may not be fulfilled. In this case, our analysis of the numerical simulation carried out by Chen [16] reveals that a proportionality $\dot{m} \propto$ $\delta_{s} v U \rho_{s \infty} / \alpha_{s}$ may better represent the mass flux of volatiles for large values of opposed flow velocities. In this case, we would expect a higher dependence of the flame spread rate on $U$ than that obtained from Eq. (49).

We point out that, here, buoyancy effects are only taken into account through the $l$ parameter since it includes the induced flow velocity. Due to the small sensitivity of the solution to changes in $l$, we conclude that our model is almost independent of the gravity level, as also observed in Eq. (1) to which we compare with. In the downward burning of thin cellulosic-type fuels in a quiescent atmosphere (as in the case here analyzed), Kumar and Kumar have recently obtained a variation of the flame front speed below 3\% between the normal gravity (1g) and the zero gravity $(0 \mathrm{~g})$ cases (although this figure may raise to $25 \%$ when comparing the $1 \mathrm{~g}$ case with some microgravity levels) [34]. In view of these results we may accept our model as a reasonable approximation for the null background flow case.

We note, however, that even for a moderate nonzero background opposed flow $\left(15 \mathrm{~cm} \mathrm{~s}^{-1}\right)$, the flame front speed for the $0 \mathrm{~g}$ case may increase more than a $30 \%$ in comparison with the $1 \mathrm{~g}$ case [34]. In addition, buoyancy effects play a very important role in the downward combustion with concurrent background flow as well as in the upward combustion. The generalization of the present model in order to work in such regimes is not trivial, being the purpose of our current research.

On the other hand, an increase in the parameter $a$ in Eq. (27) leads to a decrease in the flame front speed $v$ in order to produce the same amount of mass flux of volatiles $\dot{m}$. Thus, for example, the velocity $v$ decreases a $52 \%$ when increasing the $a$ value from $a=0.229$ till $a=1$ for the case with Ar as a diluent, $X_{O \infty}=0.3$ and $\delta_{s} \rho_{s \infty}=0.04 \mathrm{~kg} \mathrm{~m}^{-2}$.

Equation (49) assumes that the heat flux into the solid is entirely used for vaporizing it. This overestimates the amount of volatiles since part of the heat flux will be also used for preheating the solid. Thus, it is expected that $\left|\lambda_{g} \partial T / \partial y\right|_{y=0}>$ $\dot{m} L$, so we may define a factor $w(>1)$ such as $\left|\lambda_{g} \partial T / \partial y\right|_{y=0}=$ $w \dot{m} L$. In comparison with the case $w=1$ analyzed above, the thin-solid fuel case with $\mathrm{Ar}$ as a diluent $X_{O \infty}=0.3, \delta_{s} \rho_{s \infty}=$ $0.04 \mathrm{~kg} \mathrm{~m}^{-2}$ and $w=2$ gives a flame spread rate $v 29 \%$ lower.

\section{CONCLUSIONS}

The present paper deals with wave fronts arisen from the combustion of thin solid fuels. In comparison with the combustion of gaseous fuels, these types of processes are substantially more complex and, therefore, many techniques successfully developed for gaseous fuels cannot be applied here for analytically estimating the speed of the flame front. In the combustion of thin solid fuels, however, there exists the classical analytical expression for the flame front speed (1) derived by deRis [7] in a rigourous study that assumed infinitely fast chemical kinetics. Recently, Ref. [9] has shown that Eq. (1) may essentially follow from a qualitative analysis by using a simple control volume approach for the solid phase.

In contrast, our purpose has been to derive an analytical expression of the flame front speed by focusing on the gas phase reaction-convection-diffusion equations. This requires that we (1) model the behavior of the solid phase based on the information provided by detailed numerical simulations found in the literature and (2) make use of a one-dimensional vertical flame model. This allows us to reduce the coupled system of five reaction-convection-diffusion equations to a single one-dimensional equation with a single variable (gas mixture temperature). Finally, the analytical expression for the flame spread rate is obtained after applying the technique of matching conditions for the temperature and its derivative between the two zones of our simple combustion model.

We also derive an approximated version of our analytical expression expected to be valid for a wide range of physically realistic conditions. These analytical expressions show the same qualitative behavior to changes in the main gas and solid phase parameters than Eq. (1), although quantitative differences in a sensitivity analysis may appear. More important, and in contrast with the classical Eq. (1), our expressions are a function of (1) the preexponential factor and activation energy of the combustion reaction and (2) the solid conductivity.

A comparison study with experimental data extracted from the literature under different conditions (solid thickness, oxygen concentration, gas-type diluent) reveals that our analytical expressions successfully reproduce the observed behavior, providing better results than the classical Eq. (1). In addition, our methodology may be implemented in further studies for including the effect of a nonzero opposed flow velocity of the background flow, flame leading edge not coincident with the vaporization front, etc.

\section{ACKNOWLEDGMENTS}

B.C. acknowledges the support of a FPU grant. This work has been partially funded by the Generalitat de Catalunya under Grant 2009-SGR-374, the MICINN-FEDER under Grant FIS-2009-13050, and the European Commission under Grant NEST-28192-FEPRE. 
[1] J. Fort and V. Méndez, Rep. Prog. Phys. 65, 895 (2002); J. Fort and T. Pujol, ibid. 71, 86001 (2008).

[2] D. G. Aronson and H. F. Weinberger, Adv. Math. 30, 33 (1978); R. D. Benguria and M. C. Depassier, Phys. Rev. Lett. 77, 1171 (1996); Phys. Rev. E 57, 6493 (1998); M. Abel, A. Celani, D. Vergni, and A. Vulpiani, ibid. 64, 046307 (2001).

[3] A. Kolmogorov, I. Petrovsky, and N. Piskunov, Bull. Univ. Moscow Ser. Int. A 1, 1 (1937); Y. B. Zeldovich and D. A. Frank-Kamenetskii, Acta Physicochim. USSR 9, 341 (1938); F. A. Williams, Combustion Theory (Perseus, New York, 1985); R. D. Benguria, J. Cisternas, and M. C. Depassier, Phys. Rev. E 52, 4410 (1995).

[4] J. Fort, D. Campos, J. R. González, and J. Velayos, J. Phys A: Math. Gen. 37, 7185 (2004); T. Pujol, J. Fort, J. R. González, J. R. Montoro, and M. Pelegrí, Physica A 387, 1987 (2008); T. Pujol, J. Fort, L. Montoro, and J. J. Suñol, ibid. 388, 4959 (2009).

[5] S. J. Pyne, Introduction to Wildland Fire (John Wiley, New York, 1984).

[6] A. A. Pappa, N. E. Tzamtzis, M. K. Statheropoulos, S. E. Liodakis, and G. K. Parissakis, J. Anal. Appl. Pyrolysis 31, 85 (1995); S. Liodakis, I. Antonopoulos, and T. Kakardakis, Fire Safety J. 45, 98 (2010).

[7] J. N. deRis, Twelfth Symposium (International) on Combustion, The Combustion Institute, Pittsburgh, 241 (1969); Eq. (1) is the exact solution of deRis' model derived by M. A. Delichatsios, Combust. Sci. Technol. 44, 257 (1986).

[8] See deRis' comments in F. A. Lastrina, R. S. Magee, and R. F. Mc Alevy III, Thirteen Symposium (International) on Combustion, The Combustion Institute, Pittsburgh, 935 (1971).

[9] S. Bhattacharjee, R. Ayala, K. Wakai, and S. Takahashi, Proc. Combust. Inst. 30, 2279 (2005).

[10] A. E. Frey Jr. and J. S. T'Ien, Combust. Flame 36, 263 (1979).

[11] K-K. Wu and C-H. Chen, Combust. Sci. Technol. 175, 933 (2003); 176, 1909 (2004).

[12] J. B. Greenberg and P. D. Ronney, Int. J. Heat Mass Transf. 36, 315 (1993).

[13] J. L. Torero, T. Vietoris, G. Legros, and P. Joulain, Combust. Sci. Technol. 174, 187 (2002); A. S. Rangwala, S. G. Buckley, and J. L. Torero, Combust. Flame 152, 401 (2008).

[14] B. D. Spalding, Fourth Symposium (International) on Combustion, The Williams and Wilkins Co., Baltimore, 847 (1953).

[15] H. W. Emmons, Z. Angew. Math. Mech. 36, 60 (1956).

[16] C.-H. Chen, Combust. Sci. Technol. 69, 63 (1990).

[17] C. di Blasi, Combust. Flame 97, 225 (1994).
[18] A. Fernandez-Pello and F. A. Williams, Combust. Flame 28, 251 (1977).

[19] M. A. Delichatsios, Combust. Flame 135, 441 (2003).

[20] See, for example, R. D. Benguria, M. C. Depassier, and M. Loss, Eur. Phys. J. B 61, 331 (2008) and the works of Benguria and Depassier cited in [2].

[21] S. S. Novikov and Yu. S. Ryazantsev, J. Appl. Mech. Tech. Phys. 6, 28 (1965).

[22] D. Campos, J. E. Llebot, and J. Fort, J. Phys. A: Math. Gen. 37, 6609 (2004).

[23] S. Theodorakis and E. Svoukis, Phys. Rev. E 68, 027201 (2003).

[24] S. Martin, Tenth Symposium (International) on Combustion, The Combustion Institute, Pittsburgh, 877 (1965).

[25] C-H. Chen and J. S. T'Ien, Combust. Sci. Technol. 50, 283 (1986).

[26] As an example, for an oxygen molar fraction $X_{O 2}=20 \%$, values for the preexponential factor in Table I are $B=3.47 \times 10^{7}$ $\mathrm{m}^{3} \mathrm{~kg}^{-1} \mathrm{~s}^{-1}$ and $B=3.13 \times 10^{9} \mathrm{~m}^{3} \mathrm{~kg}^{-1} \mathrm{~s}^{-1}$ (both are $2.5 \%$ smaller only for the $X_{O 2}=50 \%$ case). In comparison: $B=1.9 \times 10^{9} \mathrm{~m}^{3} \mathrm{~kg}^{-1} \mathrm{~s}^{-1}$ for $X_{O 2}=19 \%$ [A. I. Karpov, A. A. Galat, and V. K. Bulgakov, J. Eng. Phys. Thermophys. 80, 536 (2007)], $B=1.76 \times 10^{9} \mathrm{~m}^{3} \mathrm{~kg}^{-1} \mathrm{~s}^{-1}$ for $X_{O 2}=20 \%$ [M. A. Delichatsios, Proc. Combust. Inst. 31, 2749 (2007)], $B=1.58 \times 10^{9} \mathrm{~m}^{3} \mathrm{~kg}^{-1} \mathrm{~s}^{-1}$ for $X_{O 2}=25 \%$ [C. Kumar and A. Kumar, Combust. Sci. Technol. 182, 1321 (2010) ], $B=$ $3.47 \times 10^{7} \mathrm{~m}^{3} \mathrm{~kg}^{-1} \mathrm{~s}^{-1}$ for $X_{O 2}=20 \%$ [R. A. Altenkirch, R. Eichhorn, and P. C. Shang, Combust. Flame 37, 71 (1980)].

[27] S. Bhattacharjee, J. West, and R. A. Altenkirch, Twenty-Sixth Symposium (International) on Combustion, The Combustion Institute, Pittsburgh, 1477 (1996).

[28] E. W. Lemmon and R. T. Jacobsen, Int. J. Thermophys. 25, 21 (2004).

[29] R. B. Bird, W. E. Stewart, and E. N. Lightfoot, Transport Phenomena (John Wiley, New York, 1992).

[30] T. Pujol and B. Comas, Combust. Sci. and Tech. (to be published)

[31] Y. Zhang, P. D. Ronney, E. V. Roegner, and J. B. Greenberg, Combust. Flame 90, 71 (1992).

[32] F. A. Lastrina, R. S. Magee, and R. F. Mc Alevy III, Thirteen Symposium (International) on Combustion, The Combustion Institute, Pittsburgh, 935 (1971).

[33] M. K. Wolverton, R. A. Altenkirch, and L. Tang, Combust. Flame 118, 281 (1999).

[34] C. Kumar and A. Kumar, Combust. Sci. Technol. 182, 1321 (2010). 